\title{
Main Parameter Design of Disc Milling Cutter for Machining Equal-Base Circle Bevel Gear
}

\author{
Bin Wang $\mathbb{D},{ }^{1,2}$ Kai Xu $\mathbb{D}^{\mathbb{D}},{ }^{3}$ Shuo Chen, ${ }^{1}$ Xinfeng Duan $\mathbb{D}^{1},{ }^{1}$ and Shutao Zhang ${ }^{1}$ \\ ${ }^{1}$ School of Mechatronics Engineering, Henan University of Science and Technology, Luoyang, China \\ ${ }^{2}$ Collaborative Innovation Centre of Machinery Equipment Advanced Manufacturing of Henan Province, Luoyang, China \\ ${ }^{3}$ School of Mechatronics Engineering, Zhengzhou University of Industrial Technology, Zhengzhou, China
}

Correspondence should be addressed to Kai Xu; x62025@126.com

Received 28 October 2021; Revised 10 December 2021; Accepted 27 December 2021; Published 18 January 2022

Academic Editor: Guoda Chen

Copyright (C) 2022 Bin Wang et al. This is an open access article distributed under the Creative Commons Attribution License, which permits unrestricted use, distribution, and reproduction in any medium, provided the original work is properly cited.

According to the theory and machining principle with disc milling cutter of the equal-base circle bevel gear, the mathematical model of disc milling cutter is established, the principle of machining equal-base circle bevel gear with disc milling cutter is analyzed, and the design methods of axial section profile and the parameters of disc milling cutter are studied, respectively. The reason of interference between the disc milling cutter and tooth surface during machining is studied, based on the tool motion analysis. According to the coordinate change of cutter axial section in the cutter rotation movement and the corresponding rule of coordinate between the cutter surface point and the corresponding point on tooth surface, the internal interference mechanism is analyzed. For the overcut interference in machining movement, a judgment method is proposed based on the rotation and comparison of cutter axial profile coordinates. Finally, through the processing experiment, the reasonable selection method of design parameters and the validity of judgment method are verified.

\section{Introduction}

In power generation, shipping, mining, and other important fields, super large bevel gear is the core component of large equipment. Among these key equipment fields, the super large bevel gear is mainly straight bevel gear $[1,2]$. Compared with spiral bevel gears, some special features of straight bevel gears seriously restrict the improvement and industrial upgrading of main equipment, such as poor transmission stability, large impact, high noise, and weak bearing capacity [1-4].The machine tool for machining equal-base circular gears is simple and the tool cost is low. The gear and pinion are machined by finger milling cutter on ordinary machine tool. The gear is an ideal curve bevel gear instead of straight bevel gear [5].

In the field of equal-base circle bevel gear, Cai et al. [5] proposed the concept of equal-base circle bevel gear and the method of CNC (computer numerical control) forming with finger-shaped milling cutter and verified the feasibility of $\mathrm{CNC}$ machining through experiments. Cui et al. [6] deduced the offset curves equation of the tooth line and analyzed the geometric structure characteristics, machining methods, and tool design of the equal-base circle bevel gear. Gong et al. $[7,8]$ made a deep and systematic study on the transmission principle, tooth surface geometry, tooth shape error, tooth modification, and meshing characteristics of the equal-base circle bevel gear by using modern calculation methods. Zhang et al. [9] carried out the analysis and research on the meshing characteristics, tooth surface geometry, and modification of the equal-base circle bevel gear. Zhang et al. [10] researched deeply on the theory of two-axis CNC machining of equal-base circle bevel gear. On this basis, a large size of equal-base circle bevel gear was machined with finger milling cutter.

All the above researches on the equal-base circle bevel gear are based on the finger milling cutter. Although the cost of the finger milling cutter is low, its machining efficiency is low, and the diameter is very different between the tip and the root of finger milling cutter, which leads to a large difference in the linear speed; this makes it difficult to 
control the quality of the tooth surface [2]. With the rapid development of computer technology, great progress has been made in gear advanced manufacturing [11-14], precision measurement [15], error compensation, and other aspects [16-18], especially the development of five-axis CNC machining technology [19-23], which makes it possible to machine the equal-base circle bevel gear effectively with a disc milling cutter.

Based on the above factors, the research team of authors has carried out a long-term research and put forward a new method for machining the equal-base circle bevel gear with a disc milling cutter. The derivation and calculation of CNC machining trajectory are completed, and the feasibility is verified by machining experiments [24]. It should be noted here that this method belongs to the forming method for machining gears. Due to the particularity of equal-base circle bevel gear and the novelty of this machining method, the existing gear cutting tools cannot be used for the machining; the disc milling cutter used in this method needs special design. In particular, due to the existence of the spiral angle, if the cutter diameter parameter is selected too large, it will lead to the interference between the disc milling cutter and the tooth surface. If the tool diameter is too small, the tool strength, the manufacture, and installation of the tool bar will be affected. How to design this disc milling cutter reasonably is one problem that must be solved. Therefore, this paper studies the design method, design steps, and key design points of the disc milling cutter in detail and puts forward the overcutting interference inspection method, which provides the basis for the design of the disc milling cutter.

For this purpose, the paper is organized as follows. The "Introduction" explains the background and significance of the paper briefly. The "Gear Machining Principle" describes the principle of machine the equal-base circle bevel gear with a disc milling cutter. In the "Design of Disc Milling Cutter", the design method of cutter main parameters is introduced in detail, and the calculation formula is deduced. Next, "Reasonable Design of Diameter" analyzes the internal mechanism of the interference between the cutter and the tooth surface and proposes an interference judgment method to judge the rationality of the diameter parameter. The paper is concluded in "Conclusions."

\section{Gear Machining Principle}

In order to understand the subsequent tool design and tool interference verification, it is necessary to briefly introduce the principle of machining equal-base circle bevel gear with disc milling cutter. This paper focuses on the design of special tool suitable for this machining principle, and the detailed machining theoretical analysis of tooth surface is referred in $[7,8]$.

According to the theory of equal-base circle bevel gear, the machining coordinate system as shown in Figure 1 is developed. When the disc milling cutter moves from the outer to the inner at a certain speed, the wheel billet rotates at different speed according to the specific rule, which makes the origin $O_{n}$ of the tool auxiliary coordinate

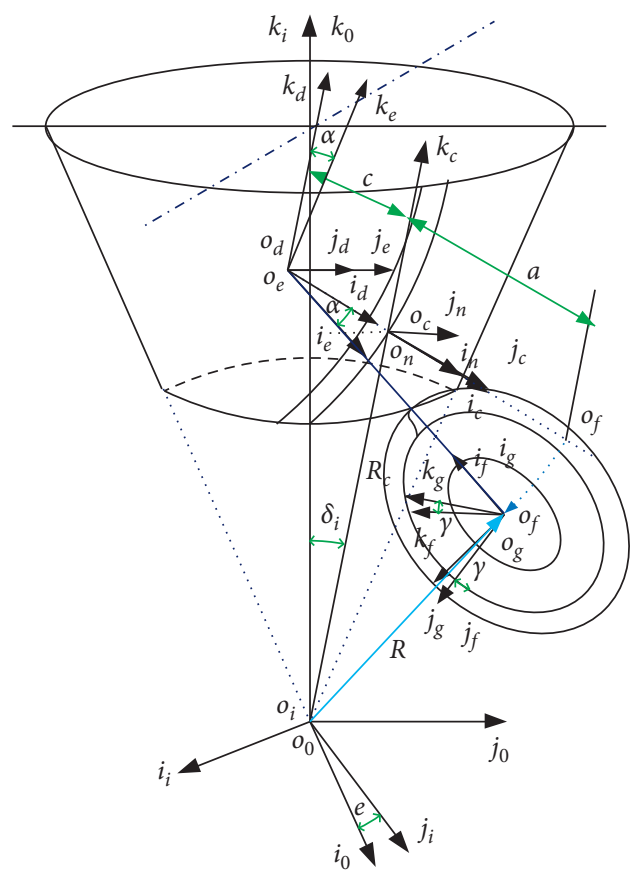

FIGURE 1: Machining coordinate system with disc milling cutter.

system $\sigma_{n}$ always be on the equidistant line of the reference cone tooth trace. In order to make the instantaneous conjugate curve of the cutter and tooth surface close to the theoretical conjugate curve, the cutter tilts the angle $\alpha$ around the axis $j_{d}$, according to the different instantaneous cutter position. At the same time, the tool is tilted angle $\gamma$ around the axis $i_{e}$; the size of $\alpha$ and $\gamma$ varies according to the helix angle $\beta$.

The coordinate systems in Figure 1 are described as follows. In the fixed coordinate system $\sigma_{o}\left(i_{o}, j_{o}, k_{o}\right)$, the origin $O_{o}$ coincides with the origin $O_{i}$ of the wheel blank coordinate system, during tooth cutting; $O_{n}$, the origin of the auxiliary coordinate system $\sigma_{n}$, is always within the plane of $i_{\mathrm{o}} j_{\mathrm{o}} k_{\mathrm{o}}$. The coordinate system $\sigma_{i}\left(i_{\mathrm{i}}, j_{\mathrm{i}}, k_{\mathrm{i}}\right)$ is fixed on the gear billet, whose origin is on the top of the cone, and the outer of theoretical tooth line is in the plane $i_{i} o_{i} k_{i} . \sigma_{c} i_{c}, j_{c}, k_{c}$ is the transitional coordinate system; the origin $O_{c}$ is always coincident with the point $O_{n}$ on the axis section profile. The intermediate coordinate system $\sigma_{d} i_{d}, j_{d}, k_{d}$ is obtained by moving the transition coordinate system $\sigma_{c} i_{c}, j_{c}, k_{c}$ a distance $c$ along the axis $i_{c}$. The tilted coordinate system $\sigma_{e} i_{e}, j_{e}, k_{e}$ is obtained by rotating the middle coordinate system $\sigma_{d}$ an angle $\alpha$ around the axis $j_{d} . \sigma_{f} i_{f}, j_{f}, k_{f}$ is the directional coordinate system of disc milling cutter; it is the result of $\sigma_{e} i_{\mathrm{e}}, j_{\mathrm{e}}, k_{\mathrm{e}}$ moving a distance $a-c$ along axis $i_{e}$ and rotating. $\sigma_{g} i_{g}, j_{g}, k_{g}$ is the coordinate system of disc milling cutter, which is obtained by rotating the coordinate system $\sigma_{f}$ an angle $\gamma$ around $i_{f}$.

Define the transformation matrix as follows: $\sigma_{c} \longrightarrow \sigma_{o}$ : $\mathbf{M}_{o c} ; \sigma_{o} \longrightarrow \sigma_{i}: \mathbf{M}_{i o} ; \sigma_{c} \longrightarrow \sigma_{d}$ : only with displacement of $c$; $\sigma_{d} \longrightarrow \sigma_{e}: \mathbf{M}_{e d} ; \sigma_{e} \longrightarrow \sigma_{f}:$ displacement of a distance, $a-c$; then rotate $\mathbf{M}_{f e} ; \sigma_{f} \longrightarrow \sigma_{g}: \mathbf{M}_{g f}$. When inverse transformation is involved, matrices need to be inverted. Detailed transformations will not be included. 


\section{Design of the Disc Milling Cutter}

The design of disc milling cutter includes the design of profile, diameter, thickness, inner hole, keyway, and cutting edge geometric parameters. In these parameters, the design of the axial section profile, diameter, and thickness is different from that of general milling cutters, which is the focus of this section. The design of inner hole, keyway, and cutting edge parameters is the same as that of the standard disc milling cutter.

3.1. Axial Section Profile Design. The axial section profile of the finger-shaped milling cutter, used to machine the equalbase circle bevel gear, should be designed according to the tooth profile of its equivalent spur cylindrical gear. The tooth profile described here is the tooth profile of the equivalent spur cylindrical gear at the midpoint of the tooth length. As shown in Figure 2, the origin of the coordinate system $\sigma_{a}:\left[o_{a}-i_{a}, j_{a}\right]$ is the center of the equivalent gear, and the tool axis is the symmetry line between teeth. The origin of tool auxiliary coordinate system $\sigma_{n}:\left[o_{n}-i_{n}, j_{n}\right]$ is the intersection of tool axis and equivalent gear reference circle. As shown, $A B$ is a curve, $C D$ is the involute of equivalent tooth profile, and $B C$ is contacting line between $A B$ and $C D$. Tooth profile modification is realized by axial section profile modification, and the tool profile is modified in parabola form. The modification center is selected on the reference circle of equivalent gear, and the modification quantity is measured in the circumferential direction and only for the segment above $\mathrm{E}$ point. The profile of the modified tool is $A B C E D$, and the modification quantity $s_{\mathrm{k}}$ at radius $r_{\mathrm{k}}$ is

$$
s_{k}=\frac{s_{k 0}}{l_{k 0}^{2}}\left(r_{v}-r_{k}\right)^{2}
$$

where $l_{k 0}$ is the distance from the modification reference point to the modification center point, $s_{k 0}$ is the modification amount at $l_{k 0}$, it is determined by meshing analysis, and $r_{v}$ is the reference circle radius of equivalent gear.

In the working part of the tooth profile in coordinate system $\sigma_{a}$, the equation of the $\mathrm{CD}$ segment is

$$
\left\{\begin{array}{l}
x_{k}=r_{k} \cos \left(\tau_{k}+\frac{s_{k}}{r_{k}}\right), \\
y_{k}=-r_{k} \sin \left(\tau_{k}+\frac{s_{k}}{r_{k}}\right),
\end{array}\right.
$$

where $\tau_{k}=\pi / 2 z_{v J}-\operatorname{inv} \alpha_{n}+\operatorname{inv} \alpha_{k}$ and $r_{k}=r_{v b} / \cos \alpha_{k}$.

In the above equation, $z_{v J}$ indicates the tooth number of the equivalent gears at cone distance $R_{J}, \alpha_{n}$ indicates the pressure angle in normal plane of tooth profile, $r_{v b}$ is the base circle radius of equivalent gear, and $\alpha_{k}$ is an independent variable as shown in Figure 2. The design of $\alpha_{k}$ should follow $\alpha_{k} \geq \alpha_{c}$; the meaning and solution of $\alpha_{c}$ are referred to in [5-8].

After the segment $C D$ is obtained, the arc $A B$ and line $B C$ are easy to obtain, so we can get the whole profile of the cutter. The axial section profile $A B C E D$, rotates around the

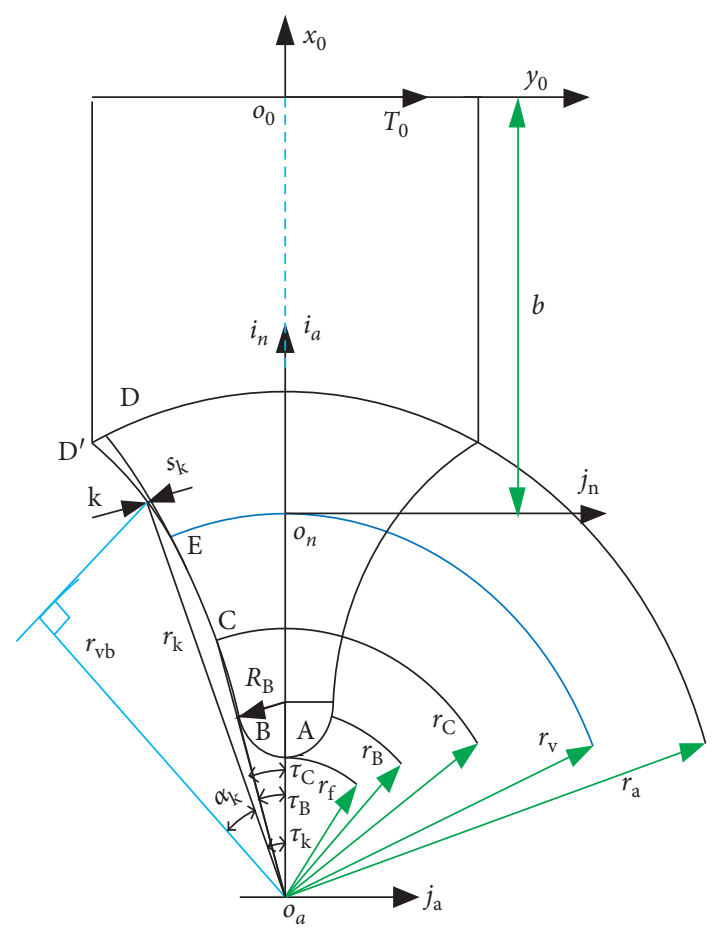

FIgURE 2: Design of the milling cutter.

axis $y_{0}$ in Figure 2 to form a disc milling cutter, and $y_{0}$ is $b$ away from and parallel to $j_{n}$; the tool diagram is shown in Figure 3. Figure 3 is only the outline diagram of the tool. In practice, the cutting edge and tool body are made separately and then assembled, which is more reasonable.

3.2. Design of Tool Thickness. When the disc milling cutter is used to machine the equal-base circle bevel gear, it must be ensured that the cutter can pass through the inner teeth space without scratching the tooth flank of the other side. Therefore, the design of the arc tooth thickness at the reference circle should be less than the inner tooth space. That is, the cutter should be designed according to the tooth profile of the equivalent gear at cone distance $R_{J}<R_{i}$, and $R_{i}$ is the inner cone distance. According to the principle of equal-base circle bevel gear, the parameters of the equivalent gear at the design cone distance $R_{J}$ are

$$
\begin{aligned}
m_{n J} & =m_{t e}\left(R_{J} / R_{e}\right)^{3 / 2} \cos \beta_{e}, \\
z_{v J} & =\frac{z}{\left(R_{J} / R_{e}\right)^{3 / 2} \cos \delta \cos ^{3} \beta_{e}}, \\
r_{v J} & =0.5 m_{n J} z_{v J},
\end{aligned}
$$

where $Z$ is the tooth number, $\delta$ is the reference cone angle, $R_{e}$ indicates the outer cone distance, $m_{t e}$ indicates the transverse module at cone distance $R_{e}$, and $\beta_{e}$ is the tooth trace spiral angle at $R_{e}$.

The cutter axial section profile is designed according to the tooth space of equivalent gear at position $R_{I}$, the half tooth thickness of the reference circle is $r_{0}=\pi m_{n J} / 4$, 


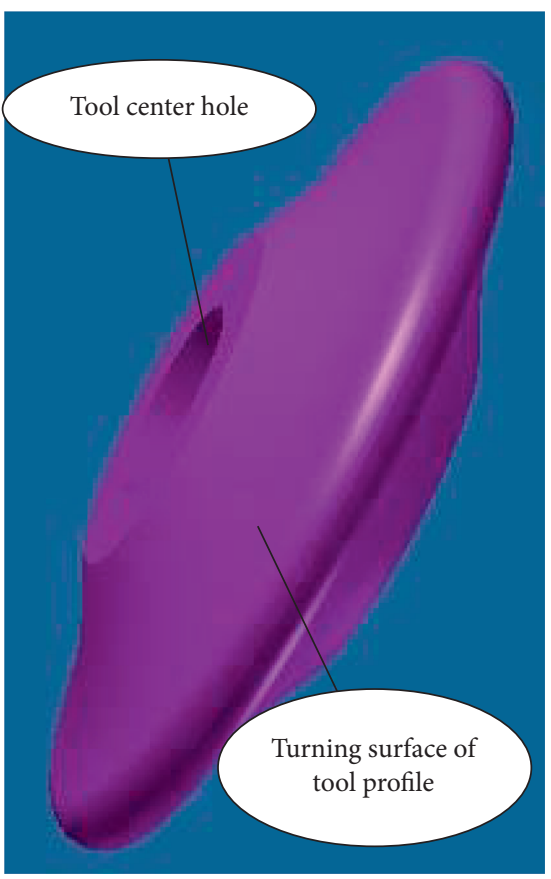

FIgURE 3: Shape of disc milling cutter.

and $r_{0}$ is the distance between the theoretical tooth trace and its equidistant line in the crown gear plane. After the tooth thickness of the reference circle is determined, the thickness in the cutting edge is obtained. As for the cutter body thickness, it can be designed to be slightly thicker to enhance the strength and rigidity of the cutter body.

3.3. Design of Cutter Diameter. As shown in Figure 2, the distance between the axes $y_{0}$ and $j_{n}$ is b. Since $y_{0}$ is the axis of disc milling cutter, obviously the diameter of disc milling cutter depends on the design of parameter $b$.

Since the disc milling cutter is installed on the cutter arbor, and the torque is transmitted by the connecting key; therefore, it is necessary that the cutter arbor must reach a certain diameter. The cutter arbor diameter can refer to the design method of standard gear disc milling cutter. After the minimum diameter of the cutter arbor is obtained, and then the parameter $b$ is selected, its minimum value should be able to meet the installation needs of the milling cutter arbor.

It seems that the maximum value of $b$ can be arbitrarily selected, but it is not. It is found that there is a maximum value for parameter $b$; when the cutter diameter is too large, the cutter will overcut the tooth flank.

The mechanism of overcut interference and the reasonable design of parameter $b$ are complex, which is a key point in the following content of this paper. After getting the maximum and minimum values of the parameter $b$, it is reasonable in theory to take any value in this range. However, considering the strength of the disc milling cutter and the rigidity of the cutter bar, the parameter $b$ should be close to the maximum value.
3.4. Design of Other Parameters. In addition to the above three main parameters, the design of disc milling cutter also includes the design of the tool inner hole, keyway, and cutting edge geometric parameters. The design of these parameters is the same as that of general disc milling cutter used in gear machining by forming method; the specific content can be seen in the tool design manual, limited to the space, not introduced here. Among them, the design of the central hole and end face keyway is determined according to the transmission torque.

3.5. Mathematical Model of the Disc Milling Cutter. In order to analyze the interference, it is necessary to establish the mathematical model of the turning surface formed by the cutting edge. As shown in the left figure in Figure 4, the origin of coordinate system $\sigma_{n}\left(i_{n}, j_{n}\right)$ is the intersection of XOY plane of tool coordinate system $\sigma(X, Y, Z)$ and the pitch circle of equivalent spur gear. So, set the equation of the known turning surface in the tool coordinate system as

$$
\begin{aligned}
X & =R \cdot \cos \varphi Y \\
& =R \cdot \sin \varphi Z \\
& =f(R) .
\end{aligned}
$$

Combined with the profile design of the disc milling cutter, the equation of the cutter surface can be obtained as follows:

$$
\left\{\begin{array}{l}
X=\left(-r_{k} \cos \left(\tau_{k}+\frac{s_{k}}{r_{k}}\right)+r_{v}+b\right) \cdot \cos \varphi, \\
Y=\left(-r_{k} \cos \left(\tau_{k}+\frac{s_{k}}{r_{k}}\right)+r_{v}+b\right) \cdot \sin \varphi, \\
Z= \pm r_{k} \sin \left(\tau_{k}+\frac{s_{k}}{r_{k}}\right),
\end{array}\right.
$$

where " + " indicates a right-handed convex and left-handed concave tooth surfaces and " - " indicates a right-handed concave and left-handed convex tooth surfaces. $b$ is the distance from axis of the plate-shape gear cutter to the origin $O_{n}$ of the auxiliary coordinate system $\sigma_{n}(\mathrm{~mm})$; and $\varphi$ is the angle between radius $R$ and plane XOZ; the positive direction is from $X$ to $Y$.

\section{Reasonable Design of Diameter}

After the basic parameters design of the disc milling cutter, another key of tool design is to determine the rationality of diameter parameters, which is directly related to the interference of overcutting. Therefore, the internal mechanism between the tool diameter and interference is studied firstly in this section, and then a judgment method is proposed.

4.1. Tool Rotation Analysis. In order to explore the causes of overcut, it is necessary to analyze the rotation of the disc milling cutter firstly, so as to establish the rotation analysis 


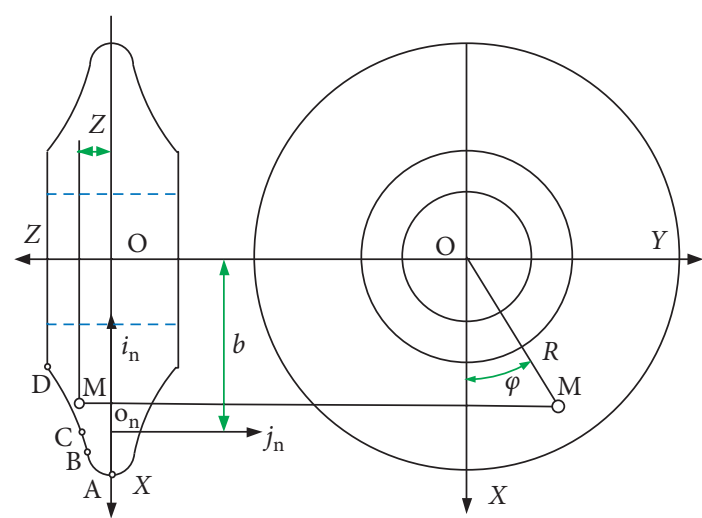

FIgURE 4: Model of disc milling cutter.

model of the cutter as shown in Figure 5. In coordinate system $\left(x^{\prime}, y^{\prime}, z^{\prime}\right)$, the axis of disc milling cutter coincides with axis $z^{\prime}$. Coordinate origin $o^{\prime}$ is the center point of cutter axis profile, and the direction of each axis is as illustrated in the figure. The $\sigma_{n}:\left(i_{n}, j_{n}\right)$ is the auxiliary coordinate system for axial section profile of the cutter, in which the axial section profile coordinates are $x_{n}$ and $y_{n}$ and the distance from origin $o_{n}$ to $o^{\prime}$, the origin of disc cutter coordinate system, is $b$, whose value determines the diameter of the cutter. Points $o^{\prime}\left(o_{1}\right)$ and $o_{2}$ are the rotary center of the smaller and the bigger diameter cutter, respectively. Arbitrarily, pick up a point $A_{0}$ in the profile of smaller diameter cutter; in the process of cutter rotating around $o_{1}$, point $A_{0}$ turns to $A_{1}$ and rotation angle is $\alpha$. Pick up the same point $A_{0}$, which is in the profile of bigger diameter cutter; in the process of cutter rotating around $o_{2}$, point $A_{0}$ turns to $A_{2}$ and rotation angle is still $\alpha$. The plane where point $A_{0}$ rotates is parallel to coordinate plane $x^{\prime} o^{\prime} y^{\prime}$, the distance from the origin is $z=-y_{n}$, and the coordinates of $A_{1}$ in Figure 5 can be easily obtained.

$$
\left\{\begin{array}{l}
x_{1}^{\prime}=\left(-x_{n}+b\right) \cdot \cos \alpha \\
y_{1}^{\prime}=-\left(-x_{n}+b\right) \cdot \sin \alpha \\
z_{1}^{\prime}= \pm y_{n}
\end{array}\right.
$$

In the rotation, the point raised a length $A_{0} A_{4}$ in the direction of $x^{\prime}$ :

$$
A_{0} A_{4}=\left(-x_{n}+b\right)-\left(-x_{n}+b\right) \cdot \cos \alpha .
$$

The point moves a distance $A_{1} A_{4}$ in the direction of $y^{\prime}$ :

$$
A_{1} A_{4}=\left(-x_{n}+b\right)-\left(-x_{n}+b\right) \cdot \sin \alpha .
$$

In the same way, the coordinates of point $A_{2}$ can be easily obtained.

4.2. Overcut on the Tooth Surface. While the contact of the cutter surface and tooth surface is in a correct state, the position relation between $\left[0 /-x^{\prime}, y^{\prime} z^{\prime}\right]$, the cutter coordinate system in Figure 5, and the wheel blank is shown in Figure 6; arbitrary point $A_{0}$ on the profile of cutter rotates around the axis $o^{\prime} z^{\prime}$ and moves to point $A_{1}$; the distance to plane $x^{\prime} o^{\prime} y^{\prime}$ is $a^{\prime}$; the tooth trace corresponding to point $A_{0}$

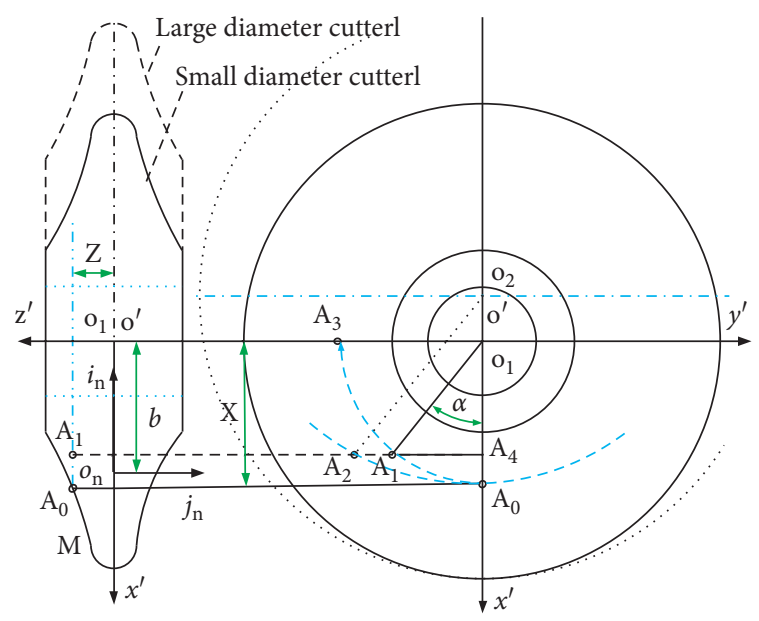

Figure 5: Analysis diagram of disc milling cutter rotation.

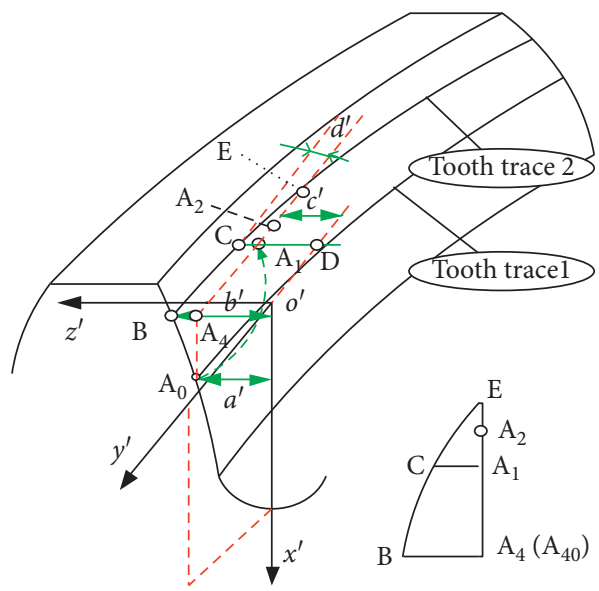

FIGURE 6: Schematic diagram of overcut on the tooth surface.

is tooth trace 1 . When point $A_{0}$ turns to point $A_{1}$, the distance $A_{0} A_{4}$, point $A_{0}$ moving in the direction $x^{\prime}$, has been obtained; the corresponding tooth trace in this height is tooth trace 2 , which is passing point $B$ on the tooth surface. The distance from point $B$ on the tooth trace 2 to plane $x^{\prime} o^{\prime} y^{\prime}$ is $b^{\prime}$; point $C$ is the intersection point of two lines; one is a straight line which is through point $A_{1}$ and perpendicular to the plane $x^{\prime} o^{\prime} y^{\prime}$ and the other is tooth trace 2; the straight line intersects with the plane $x^{\prime} o^{\prime} y^{\prime}$ at point $D ; A_{1} C$ $=d^{\prime}$ and $A_{1} D=c^{\prime}$. Obviously, in Figure $6, a^{\prime}=c^{\prime}$ and $a^{\prime}$ equals $z=-y_{n}$ in Figure 5 .

In the process of point $A_{0}$ passing point $A_{1}$ and turning to point $A_{3}$ in Figure 5 , along with the raise of $A_{0}$ in the direction of $x^{\prime}, A_{0} A_{4}$ is increased corresponding to each instant. In Figure $6, z^{\prime}$ is changing correspondingly to each instant; $A_{4} B=b^{\prime}-a^{\prime}$ can be obtained in Figure 6 .

Assume that there is no spiral angle; each of the tooth traces 1 and 2 will be straight line. Because $b^{\prime}$ is always larger than $a^{\prime}$, when an arbitrary point $A_{0}$, which is on the axis section profile of the cutter, rotates to point $A_{1}$, there must be a certain distance to tooth surface; thus interference will not appear; the distance is $d^{\prime}, d^{\prime}=b^{\prime}-a^{\prime}$; it can be obtained 
by the figure. As a result of the existence of spiral angle, as shown in the bottom right corner of Figure 6, when point $A_{0}$ rotates to point $A_{1}, A_{1} C$ must be less than $A_{4} B$ this moment; that is to say, $d^{\prime}$ is less than $b^{\prime}-a^{\prime}$.

This moment, whether the interference appears or not depends on the value of $d^{\prime}\left(A_{1} C\right)$; when $d^{\prime}=0$, point $\mathrm{C}$ coincides with point $A_{1}$; while the value of $d^{\prime}$ is less than 0 , point $A_{1}$ passes through point $C$ and enters the tooth surface; then the interference appears. In Figure 5, when larger diameter cutter is used, the rotary center of the cutter is in point $o_{2}$; while it raised the same distance $A_{0} A_{4}$ in the direction of $x^{\prime}$, the moving distance of point $A_{0}$ in the direction of $y^{\prime}$ is

$$
A_{2} A_{4}=\sqrt{o_{2} A_{2}^{2}-o_{2} A_{4}^{2}}
$$

where $o_{2} A_{2}=o_{2} A_{0}$, where $o_{2} A_{0}$ is known; obviously $A_{2} A_{4}$ is longer than $A_{1} A_{4}$ in Figure 5. And, we can see in Figure 6 that, with the increase of cutter diameter, point $A_{2}$ tends to point $E$; while reaching point $E$, the inference occurs between the cutter and the tooth surface.

So, there are two causes of interference occurring: on the one hand, when spiral angle is fixed, due to the increasing of the cutter diameter, point $A_{1}$ reaches point $A_{2}$ and tends to point $E$ and the interference occurs. On the other hand, when the cutter diameter is fixed, the changing rate of spiral angle is too large, and the value of $d^{\prime}$ is less than 0 . While point $A_{0}$ rotates to point $A_{1}$, the inference occurs. Figure $7(\mathrm{a})$ is the precise simulation modeling of the cutting surface and theoretical tooth surface of the tool by MATLAB in a certain machining position. It can be seen clearly that the tool has been embedded in the theoretical tooth surface. Figure $7(\mathrm{~b})$ is a three-dimensional modeling of Inventor software under the same condition, and the overcut interference in the figure is more intuitive. The overcut interference shown in Figure 7 occurs during the machining of the concave tooth surface. For the convex tooth surface, because the cutter of the same size and shape is used, and the tooth line of convex surface is convex to the tooth space, there is no overcut interference, so the research on overcut interference in this paper is only for the concave tooth surface.

4.3. Overcut Judgment. According to the previous tool rotation analysis, take a number of points on the profile line in the vertical tool axis section, take the tool axis as the rotation center, rotate these points at a certain angle interval towards the inner direction of the gear, and a set of points will be obtained. In the same way, according to the above principle, the corresponding points set of tooth surface can also be obtained on the tooth surface. At this time, the two point sets are located on the tool surface and tooth surface, respectively. By comparing the coordinates of all these points, it can be determined whether the tool and tooth surface interfere at this time. If the interference occurs, change the tool diameter and continue the above analysis process.

4.3.1. Calculation of Discrete Points on the Tool Surface. The interference in the machining mainly occurs on concave tool surface. In this case, for the tooth profile points in auxiliary coordinate system $\sigma_{n}$, the vector translated to coordinate system $\sigma_{e} i_{e}, j_{e}, k_{e}$ can be expressed as $\left[X_{e}, Y_{e}, Z_{e}\right]$ $=\left(x_{n},-y_{n}, 0\right)$, as shown in Figure 1. Then, translate the vector to coordinate system $\sigma_{f} i_{f}, j_{f}, k_{f}$. According to the cutter mathematical model in Figure 4 , the coordinates of points on cutter surface in $\sigma_{f} i_{f}, j_{f}, k_{f}$ are $\left(x_{\mathrm{n}}-a,-\right.$ $\left.y_{\mathrm{n}}, 0,1\right) \cdot \mathbf{M}_{2}$ :

$$
\mathbf{M}_{2}=\left[\begin{array}{cccc}
\cos \varphi & 0 & -\sin \varphi & 0 \\
0 & 1 & 0 & 0 \\
-\sin \varphi & 0 & \cos \varphi & 0 \\
0 & 0 & 0 & 1
\end{array}\right] .
$$

When angle $\varphi$ takes different values, the points set coordinates on the tool surface can be obtained. Machining starts from the outer of the gear blank, $\varphi$ takes the negative in Figure 4 . The maximum angle can be judged by the coordinates of point $A_{1}$ in Figure 5, to make it in the range of tooth depth. After obtaining the points set on the tooth surface, convert it to the fixed coordinate system $\sigma_{\mathrm{o}} i_{\mathrm{o}}, j_{\mathrm{o}}, k_{\mathrm{o}}$ in Figure 1. According to the theory of coordinate transformation and vector resultant, the points set $\left[X_{0}, Y_{0}, Z_{0}\right]$ in fixed coordinate system $\sigma_{o}$ is

$$
\left[X_{0}, Y_{0}, Z_{0}, 1\right]=\left(x_{n}-b,-y_{n}, 0,1\right) \cdot \mathbf{M}_{2} \cdot \mathbf{M}_{g f} \cdot \mathbf{M}_{o c} .
$$

In Figure 1, the origin of coordinates system $\sigma_{g} i_{g}, j_{g}, k_{g}$ can be transformed to fixed coordinate system $\sigma_{o} i_{o}, j_{o}, k_{o}$, which can be represented as

$$
[b, 0,0,1] \cdot \mathbf{M}_{\mathrm{oc}}+\left[R_{\mathrm{c}} \cdot \sin \delta, 0, R_{\mathrm{c}} \cdot \cos \delta, 0\right] .
$$

The calculation formula of $R_{c}$ in the above is relatively long, which will not be described here; see [5] for details. Combine and simplify the vector in formulas (11) and (12); the points set vector $\left[x_{d}, y_{d}, z_{d}\right]$ on cutter surface in fixed coordinate system $\sigma_{o}$ is

$$
\left\{\begin{array}{l}
x_{d}=\left[\cos \delta \cos \varphi A_{1}-y_{k} \sin \delta \sin \gamma-\sin \delta \cos \gamma \sin \varphi A_{1}\right]+R_{c} \sin \delta+b \cos \delta, \\
y_{d}=y_{k} \cos \gamma-\sin \gamma \sin \varphi A_{1}, \\
z_{d}=\left(-A_{1} \sin \delta-y_{k} \cos \delta \sin \gamma-A_{1} \cos \delta \cos \gamma \sin \varphi\right)+R_{c} \cos -b \sin \delta,
\end{array}\right.
$$




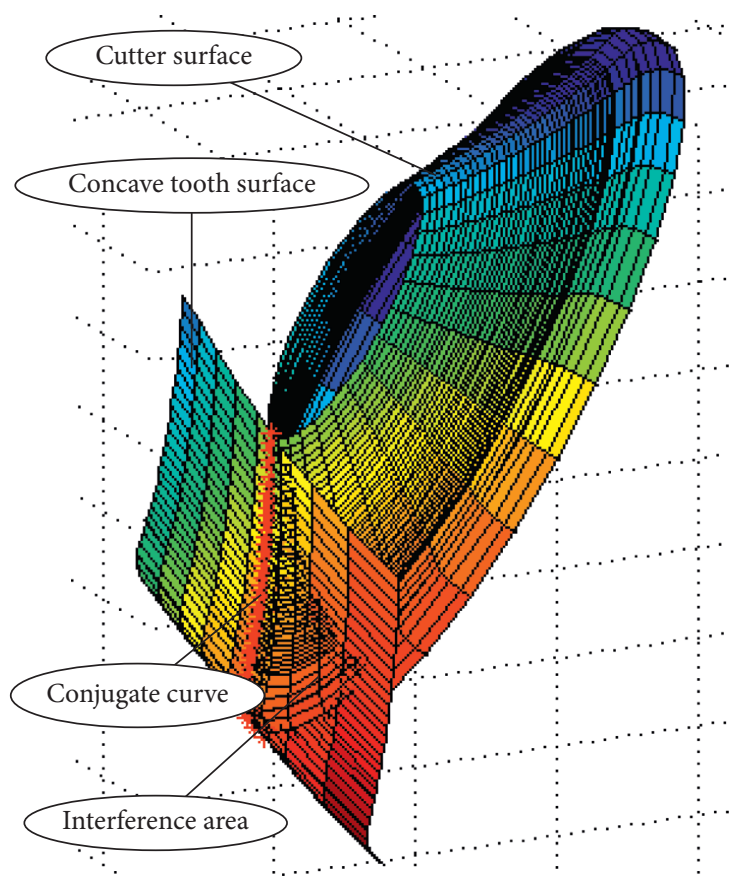

(a)

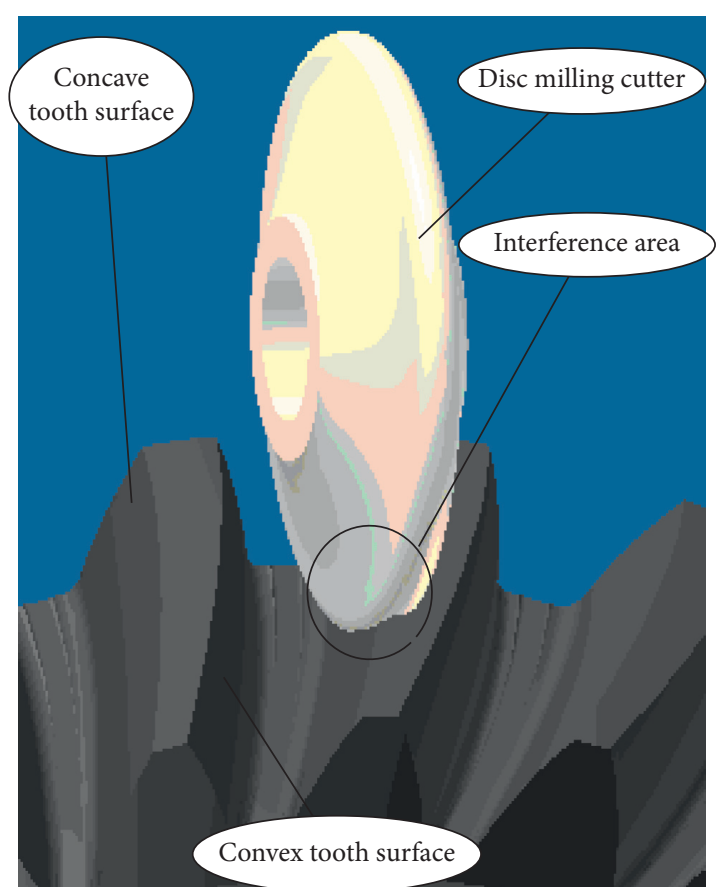

(b)

Figure 7: Interference between disc milling cutter and theoretical tooth surface. (a) Precise modeling drawing. (b) Three-dimensional schematic diagram.

where $A_{1}=\left(x_{k}-r_{v}-b\right)$.

\subsubsection{Calculation of Discrete Points on the Tooth Surface.} After obtaining the points set on the cutter face, a points set can be obtained on the tooth face, and each point in this points set corresponds to a point in the points set on the tool face. As shown in Figure 2, because the axial section profile equations of different sections are different, it is necessary to determine the range of corresponding points on the tooth surface first and then start the calculation. The specific steps are as follows.

First of all, determine the height range of tooth surface point in the direction of tooth depth. In Figure 2, the coordinate of axial section profile is easy to get in the auxiliary coordinate system $\sigma_{n}:\left[o_{n}-i_{n}, j_{n}\right]$, the coordinates of special points $A, B, C$, and $D$ are also easy to obtain, and the origin of coordinate system $\sigma_{n}$ coincides with the origin of transition coordinate system $\sigma_{c} i_{c}, j_{c}, k_{c}$ in Figure 1. Transform the vector $\left[x_{d}, y_{d}, z_{d}\right]$ on cutter surface, obtained by formula (12), to coordinate system $\sigma_{c} i_{c}, j_{c}, k_{c}$ ); if the coordinates of $x_{d}$ in the direction of axis $i_{c}$ are bigger than those of point $C$, the corresponding tooth surface point will be in $C D$ segment. If the coordinates of $x_{d}$ are between point $B$ and point $C$, the corresponding tooth surface point will be in $B C$ segment. If the coordinates of $x_{d}$ are smaller than point $B$, the corresponding tooth surface point will be in $A B$ segment. The vector $\left[x_{c}, y_{c}, z_{c}\right]$ is

$$
\begin{aligned}
{\left[x_{c}, y_{c}, z_{c}, 1\right]=} & \left\{M_{f g} \cdot\left[x_{d}, y_{d}, z_{d}, 1\right]^{T}\right\}^{T} \\
& +\left[R_{c} \cdot \sin \delta, 0, R_{c} \cdot \cos \delta, 0\right] .
\end{aligned}
$$

Secondly, the relevant calculation parameters are solved. When the point on tooth surface is in $C D$ segment, the required parameters are $\alpha_{\mathrm{k}}$ and $R$, while, in $B C$ segment, the required parameters are $\tau_{k}$ and $R$ and in $A B$ segment the required parameters are $\theta_{k}$ and $R$. According to the above judgment method, there are corresponding calculation formulas for angle parameters $\alpha_{k}, \tau_{k}$, and $\theta_{k}$. When calculating the cone distance parameter $R$ corresponding to point $A_{1}$ in Figure 6, the value of $A_{1} A_{4}$ in Figure 6 and the cone distance parameter corresponding to point $B$ are required firstly, and then $R$ can be obtained by a simple geometric calculation.

Finally, the calculation formula of discrete points on the tooth surface is derived. According to the tooth surface equation of the equal-base circle bevel gear and the parameters above, the discrete point $\left[x_{i}, y_{i}, z_{i}\right]$ in the wheel blank coordinate system can be obtained.

$$
\left\{\begin{array}{l}
x_{i}=r_{k} \cdot \cos \left(\tau_{k}+s_{k} / r_{k}\right)-r_{v}, \\
y_{i}=-r_{k} \cdot \sin \left(\tau_{k}+s_{k} / r_{k}\right) \cdot \cos \gamma, \\
z_{i}=r_{k} \cdot \sin \left(\tau_{k}+s_{k} / r_{k}\right) \cdot \sin \gamma .
\end{array}\right.
$$

After the judgment point is selected, that is to say, when the cone distance parameter is a certain value, the discrete points of the tooth surface in the wheel blank coordinate system need to be converted to the fixed coordinate system, but the conversion matrix is related to the cone distance in this case. If the cone distance is different, the angle $e$ in Figure 1 will be different. If the angle value in this case is $e_{0}$, the transformation matrix is 


$$
M_{o i}^{\prime}=\left[\begin{array}{cccc}
\sin e_{0} & \cos e_{0} & 0 & 0 \\
-\cos e_{0} & \sin e_{0} & 0 & 0 \\
0 & 0 & 1 & 0 \\
0 & 0 & 0 & 1
\end{array}\right] .
$$

Based on the above analysis, the discrete point of tooth surface in the fixed coordinate system is

$$
\begin{aligned}
r_{\mathrm{i} 2}= & \mathbf{M}_{o i}^{\prime} \cdot \mathbf{M}_{o i} \cdot \mathbf{M}_{o c} \cdot\left[x_{\mathrm{i}}, y_{\mathrm{i}}, z_{\mathrm{i}}, 1\right]^{\mathrm{T}} \\
& +\mathbf{M}_{i o} \cdot\left[R_{\mathrm{c}} \cdot \sin \delta, 0, R_{\mathrm{c}} \cdot \cos \delta, 1\right]^{\mathrm{T}} .
\end{aligned}
$$

TABLE 1: Basic geometric parameters of gear pair.

\begin{tabular}{lc}
\hline Parameter & Value \\
\hline Tooth number of pinions $Z_{1}$ & 15 \\
Tooth number of wheels $Z_{2}$ & 23 \\
Outer transverse module $M_{\text {te }}$ & 12 \\
Face width $B$ & 40 \\
Outer spiral angle $\beta_{e}$ & $6^{\circ}$ \\
Normal pressure angle & $20^{\circ}$ \\
Shaft angle $\gamma$ & $90^{\circ}$ \\
\hline
\end{tabular}

Solve and simplify formula (17); in the fixed coordinate system, the discrete points $\left[x_{c}, y_{c}, z_{c}\right]$ on tooth surface which is corresponding to discrete points of cutter surface are

$$
\left\{\begin{array}{l}
x_{0}=\sin e_{0}\left\{A_{3} \sin e+r_{k} \cos e \sin A_{1} \cos \gamma+R_{c} \sin \delta \sin e\right\}+\cos e_{0}\left\{A_{3} \cos e-r_{k} \sin e \sin A_{1} \cos \gamma+R_{c} \sin \delta \cos e\right. \\
y_{0}=-\cos e_{0}\left(A_{3} \sin e+r_{k} \cos e \sin A_{1} \cos \gamma+R_{c} \sin \delta \sin e\right)+\sin e_{0}\left(A_{3} \cos e-r_{k} \sin e \sin A_{1} \cos \gamma+R_{c} \sin \delta \cos e\right), \\
z_{0}=-\sin \delta A_{2}+r_{k} \cos \delta \sin A_{1} \sin \gamma+R_{c} \cos \delta
\end{array}\right.
$$

$$
\text { where } \quad A_{1}=\tau_{k}+s_{k} / r_{k}, \quad A_{2}=r_{k} \cos A_{1}-r_{v} \text {, }
$$
and $A_{3}=\cos \delta A_{2}+r_{\mathrm{k}} \sin \delta \sin A_{1} \sin \gamma$.

4.3.3. Overcut Judgment. In the machining, if the cutter and the tooth surface are in normal contact, the cutter surface is located on one side of the theoretical tooth surface as a whole, which is a line contact state, and the contact line is very close to the cutter's axial section curve. After the above two kinds of discrete points are obtained, the interference between the cutter and the tooth surface can be judged.

The selection principle of tool size happens when the cutting can be completed without interference; the larger the tool diameter is, the better the machining effect is. Therefore, the tool diameter is only less than the critical value actually; the optional range is very wide, so the method of drawing, observing, and judging is simple, intuitive, and practical.

In the above drawing method, as long as there is interference between the tool and the tooth surface, it indicates that the selected tool diameter is larger; modify the tool diameter parameter and continue to draw and judge until the critical point where the interference disappears is found. It should be pointed out that, in the whole direction of tooth length, interference is most likely to occur at the place with the largest curvature of tooth line, so, only interference verification is needed at this place.

4.4. Design Example. Based on the design method and key points of the cutter, the main parameters of the disc milling cutter with a certain parameter equal-base circle bevel gear are designed. According to the data points calculated by formulas (13) and (18), the design of the cutter diameter parameters is verified by drawing method.
In this example, the geometric parameters of the gear pair are shown in Table 1, and the tool design is also based on the geometric parameters of the gear.

For different tool diameter parameters, the discrete points set of tool surface and the corresponding discrete points set of tooth surface are calculated, and the threedimensional drawing of tooth surface, tool surface, and discrete points set is carried out by MATLAB.

Figure $8(\mathrm{a})$ is an interference check drawing for the cutter with larger diameter. In the figure, the red discrete points on the tooth surface have been embedded in the cutter surface, and the interference occurs. Figure $8(\mathrm{~b})$ is an interference check drawing for the smaller cutter diameter. From the observation angle in the figure, no red discrete points are found on the tooth surface, there is only one normal contact line between the cutter surface and the tooth surface, and it can be seen that there is no interference.

Figure 9 is a picture of the disc milling cutter designed and manufactured, Figure 10 is a picture of the gear machining process, and Figure 11 is the finished equal-base circle bevel gear. After machining, the gear pair is tested by rolling. Figure 12 is a rolling test platform of a gear pair, and the test result is shown in Figure 13. Figure 14 shows the measurement and analysis results of the tooth surface. In the figure, the fine solid line represents the theoretical tooth surface, the blue solid line represents the machined tooth surface, the positive number represents the machined tooth surface overcutting, and the negative number represents the machined tooth surface undercutting. As can be seen from the graph, convex surface is overcut more, concave surface is undercut more, and the error at outer is greater than that at inner. The errors in root and top parts of teeth are larger, while the errors in middle part of teeth depth are smaller. The test results show that the contact area of concave tooth surface is normal and the tooth surface machining reaches 


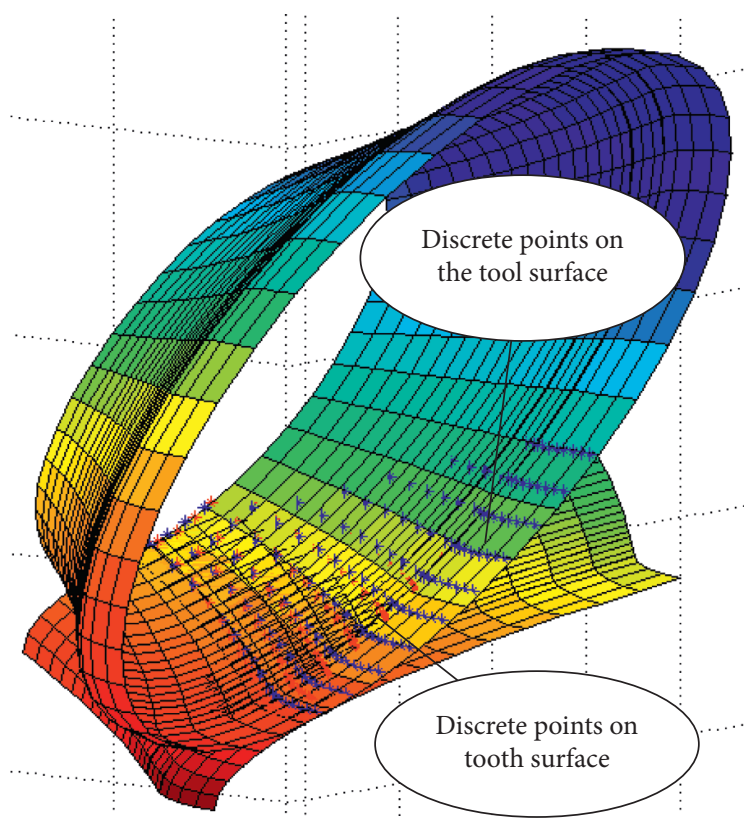

(a)

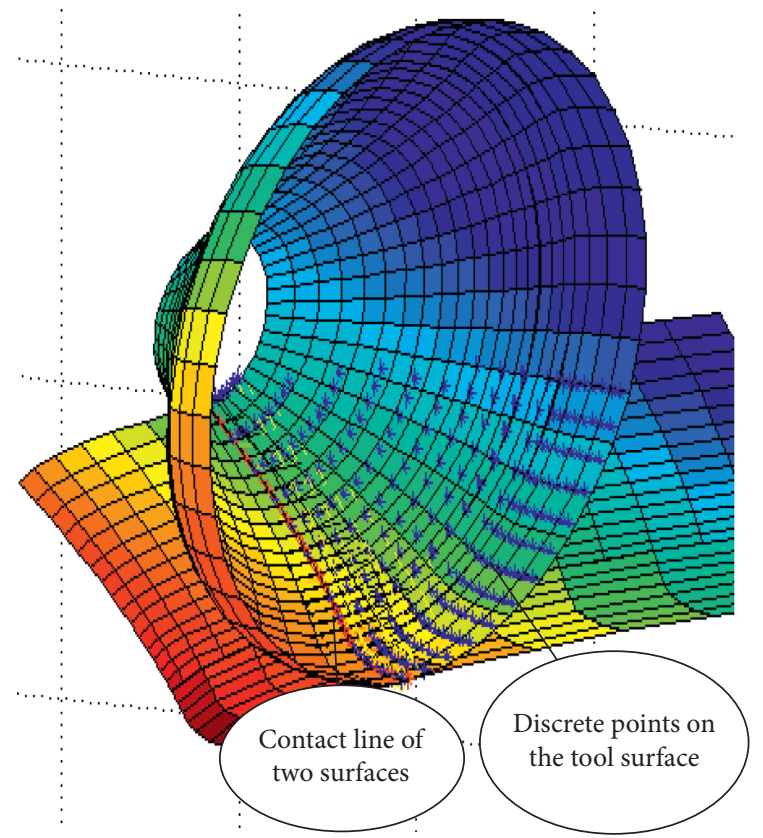

(b)

FIGURE 8: Cutter check drawings for different diameters. (a) Drawing for the larger diameter tool. (b) Drawing for the smaller diameter tool.

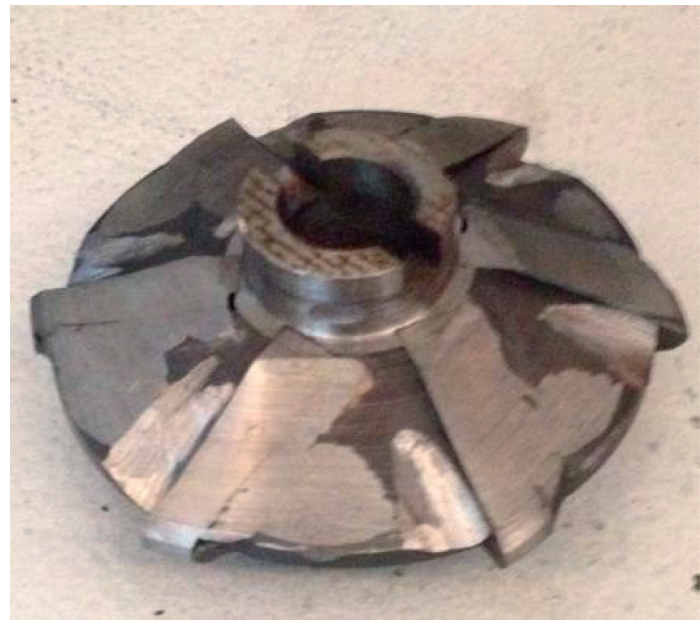

Figure 9: Picture of disc milling cutter.

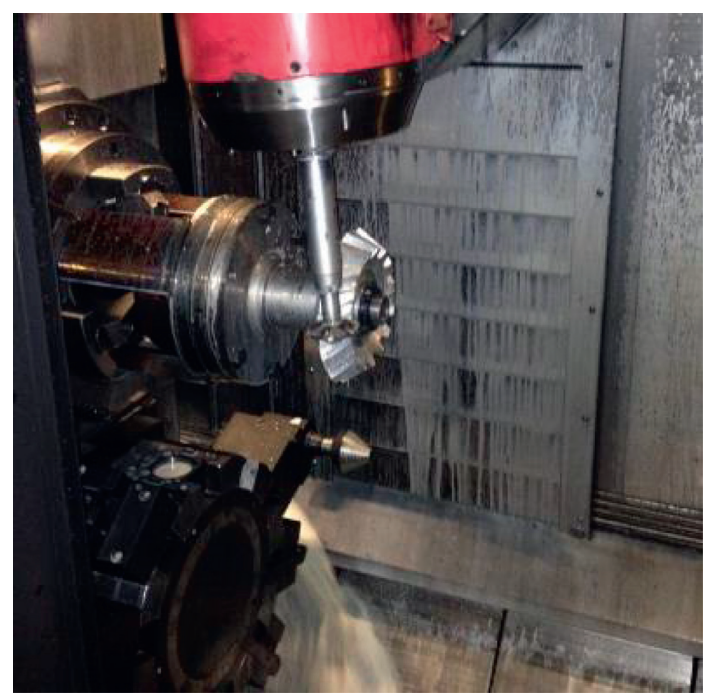

Figure 10: Photo in machining.

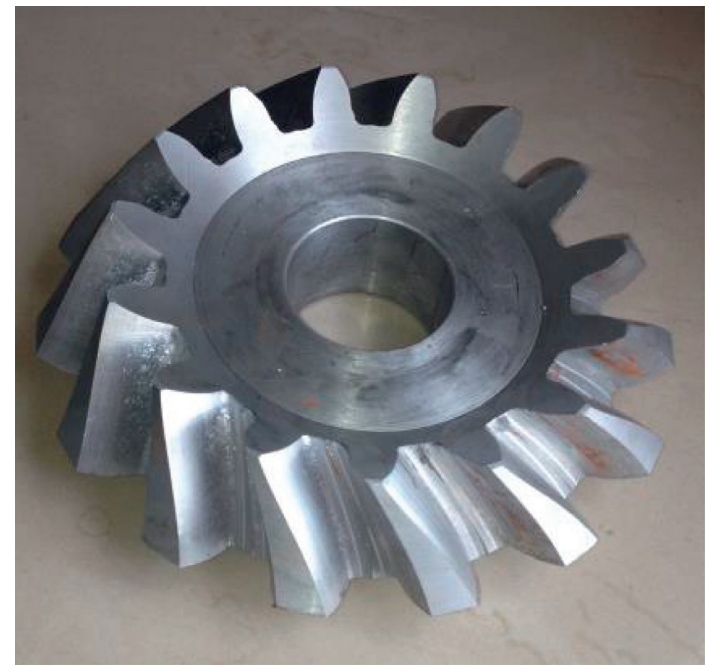

FIGURE 11: Equal-base circular gear after machining.

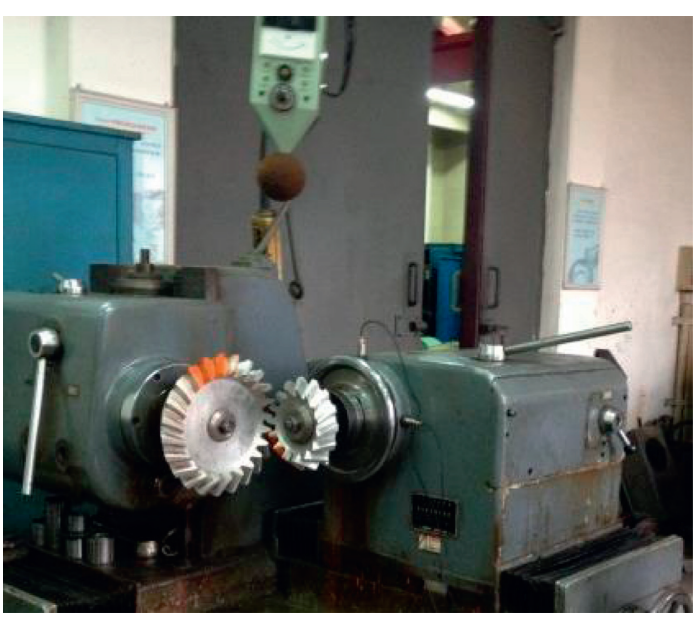

FIGURE 12: Gear rolling test platform. 


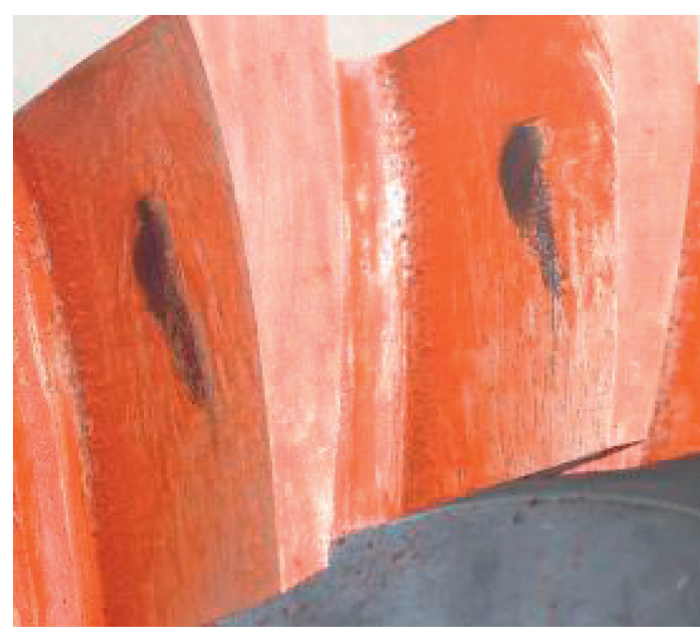

FIgURE 13: Contact area of the concave tooth surface.

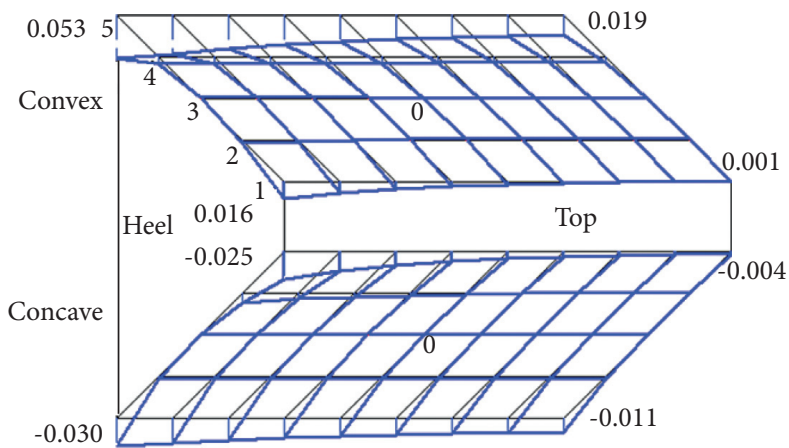

FIgURE 14: Analysis results of the tooth surface.

the expected result, which illustrates that the cutter design is reasonable and feasible.

\section{Conclusion}

A design method of the disc milling cutter is put forward for the machining of equal-base circle bevel gear, so as to realize the reasonable design of disc milling cutter. The main findings are pointed out.

The main parameters design method of the disc milling cutter, such as the curve of cutter axis section, the thickness, and the diameter of the cutter, is studied, and the calculation formulas for design parameters are obtained.

The overcut principle for tooth surface based on diameter parameter is analyzed, and a judgment method of the overcut on tooth surface based on the discrete points is proposed.

The actual tool design and gear machining experiment are carried out, and the gear machined is roll tested. The experimental results show that the design of the disc milling cutter is reasonable and the use of disc milling cutter is one of the effective methods for machining the equal-base circle bevel gear.

\section{Data Availability}

The data used to support the findings of this study are included within the article.

\section{Conflicts of Interest}

The authors declare no conflicts of interest.

\section{Acknowledgments}

This work was supported by the Key Scientific Research Projects of Colleges and Universities in Henan Province of China (no. 19A460020).

\section{References}

[1] B. Wang, X. Sun, P. Feng, C. Yan, and X. Jia, "Solution and verification of cutter position for machining split equal-base circle bevel gear," Mathematical Problems in Engineering, vol. 2019, Article ID 8024701, 14 pages, 2019.

[2] B. Wang, M. Fan, X. Sun et al., "Cutter position calculation of machining equal-base circle bevel gears with a pot-shaped milling cutter," International Journal of Advanced Manufacturing Technology, vol. 87, no. 9-12, pp. 2625-2637, 2016.

[3] I. Tsuji, K. Kawasaki, H. Gunbara, and H. Houjoh, "Tooth contact analysis and manufacture on multitasking machine of large-sized straight bevel gears with equi-depth teeth," J. Mech. Des. Trans. ASME, vol. 135, no. 3, pp. 034504:1034504:8, 2013.

[4] K. Kawasaki, I. Tsuji, H. Gunbara, and H. Houjoh, "Method for remanufacturing large-sized skew bevel gears using CNC machining center," Mechanism and Machine Theory, vol. 92, pp. 213-229, 2015.

[5] C. Cai and N. Cheng, "Principle of equal-base circle bevel gear," Chinese Journal of Construction Machinery, vol. 28, pp. 61-64, 1992.

[6] X. Cui and Y. Si, "Design of Spiral Bevel Gear with New Tooth Trace," Journal of University Pet., China, Ed. Natural Science, vol. 16, pp. 60-65, 1992.

[7] Y. Gong, W. Li, and W. Zhang, "Analysis on tooth surface curvature of equal-base-circle bevel gear," Journal of Northeast University, Natural Science, vol. 24, pp. 858-861, 2003.

[8] Y. Gong, S. Ding, and C. Cai, "Analysis of tooth form error of equal-base circle bevel gear," Journal of Mechanical Engineering of China (English edition), vol. 18, pp. 52-54, 1996.

[9] W. Zhang, Y. Gong, and D. Wang, "Analysis of tooth geometry and modification of equal-base circle bevel gear," Journal of Northeast University, Natural Science, vol. 21, pp. 390-393, 2000.

[10] B. Zhang, T. Li, and J. Chen, "Machining simulation of equalbase circle bevel gear," Journal of Henan Univerity Science Technology, Natural Science, vol. 34, pp. 18-20, 2013.

[11] C. Jiang, J. Deng, X. Deng, H. Zhang, S. Nie, and L. Geng, "Study of motional parameters calculation and tooth cutting experiment based on the new type of tilt milling machine," Journal of Advanced Mechanical Design Systems and Manufacturing, vol. 10, no. 4, pp. 15-00726:1-15-00726:19, 2016.

[12] S. X. Wu, H. Z. Yan, Z. Y. Wang, R. G. Bi, Z. Chen, and P. F. Zhu, "Tooth surface error correction of hypoid gears machined by duplex helical method," Journal of Central South University, vol. 28, no. 5, pp. 1402-1411, 2021.

[13] Y. Hou, Z. Tang, X. Shi, J. Tang, and Z. Li, “The efficient and accurate worm grinding of spur face gears according to an advanced geometrical analysis and a closed-loop manufacturing process," Journal of Central South University, vol. 29, 2022. 
[14] S. Wang, Y. Zhou, J. Tang, K. Tang, and Z. Li, "Digital tooth contact analysis of face gear drives with an accurate measurement model of face gear tooth surface inspected by CMMs," Mechanism and Machine Theory, vol. 167, pp. 104498:1-104498:20,, 2022.

[15] T. Li, J. Li, X. Deng, J. Yang, G. Li, and W. Ma, "A new digitized reverse correction method for hypoid gears based on a one-dimensional probe," Measurement Science and Technology, vol. 28, no. 12, pp. 12500:1-12500:14, 2017.

[16] J. Han, Y. Zhu, L. Xia, X. Tian, and B. Yuan, "Modeling and adaptive compensation of tooth surface contour error for internal gearing power honing," Proceedings of the Institution of Mechanical Engineers - Part B: Journal of Engineering Manufacture, vol. 233, no. 5, pp. 1500-1514, 2019.

[17] S. Xiang and Y. Altintas, "Modeling and compensation of volumetric errors for five-axis machine tools," International Journal of Machine Tools and Manufacture, vol. 101, pp. 6578, 2016.

[18] N. Huang, Y. Jin, Q. Bi, and Y. Wang, "Integrated postprocessor for 5-axis machine tools with geometric errors compensation," International Journal of Machine Tools and Manufacture, vol. 94, pp. 65-73, 2015.

[19] Y. Z. Wang, Z. Lan, L. W. Hou, H. P. Zhao, and Y. Zhong, “A generating milling method for a spur face gear using a fiveaxis computer numerical control milling machine," Proceedings of the Institution of Mechanical Engineers - Part B: Journal of Engineering Manufacture, vol. 230, no. 8, pp. 1440-1450, 2016.

[20] Y. Zhou, Z. C. Chen, J. Tang, and S. Liu, “An innovative approach to NC programming for accurate five-axis flank milling of spiral bevel or hypoid gears," Computer-Aided Design, vol. 84, pp. 15-24, 2017.

[21] X. Deng, G. Li, B. Wei, and J. Deng, "Face-milling spiral bevel gear tooth surfaces by application of 5-axis CNC machine tool," International Journal of Advanced Manufacturing Technology, vol. 71, no. 5-8, pp. 1049-1057, 2014.

[22] J. Yang and Y. Altintas, "Generalized kinematics of five-axis serial machines with non-singular tool path generation," International Journal of Machine Tools and Manufacture, vol. 75, pp. 119-132, 2013.

[23] Y. Shih and C. Zhang, "Manufacture of spiral bevel gears using standard profile angle blade cutters on a five axis computer numerical control machine," Journal of Manufacturing Science and Engineering, vol. 139, no. 6, pp. 061017:1-061017:21, 2017.

[24] J. Deng, B. Wang, X. Deng, and J. Li, "The theoretical and experimental research of machining equal-base circle bevel gears with a pot-shaped milling cutter," Jouranl of Advanced Mechanical Design System Manufacturing, vol. 9, no. 3, pp. 1-18, 2015. 